\title{
Universitat Oberta de Catalunya: la primera universidad nacida en línea Entrevista con Albert Sangrà
}

\section{Universitat Oberta de Catalunya: la première université née en ligne - Entretien avec Albert Sangrà}

\section{Universitat Oberta de Catalunya: The first university born online - Interview with Albert Sangrà}

\author{
Marcelo Maina, professor \\ Universitat Oberta de Catalunya, España \\ mmaina@uoc.edu
}

\section{RESUMEN}

Albert Sangrà es Catedrático de la Universitat Oberta de Catalunya (UOC) y director de la Cátedra UNESCO en Educación y Tecnología para el Cambio Social. Como miembro fundador, en esta entrevista nos presenta un breve recorrido de dicha universidad desde su nacimiento a mediados de los años 1990 y destaca su particularidad de ofrecer desde sus inicios una formación en lengua catalana en línea que poco tiempo después incorporaría el español. A continuación, expone los principios en los que se basa actualmente el e-learning en la UOC y, por último, expresa su opinión sobre el futuro de esta modalidad de formación a la luz del impacto de la pandemia de COVID-19.

Palabras clave: aprendizaje en línea, educación superior, modelo educativo, futuro de educación, pandemia, España, Catalunya

\section{RÉSUMÉ}

Albert Sangrà est professeur titulaire pour l'Université Ouverte de Catalogne (UOC) et directeur de la Chaire UNESCO en éducation et technologie pour le changement social. En tant que membre fondateur de cette université, il en retrace brièvement la naissance au milieu des années 1990 et souligne sa particularité d'offrir dès le départ une formation en langue catalane en ligne qui a intégré peu après l'espagnol. II expose ensuite les principes sur lesquels s'appuie actuellement l'apprentissage en ligne à l'UOC et, enfin, fait part de son point de vue sur l'avenir de ce mode de formation à la lumière de l'impact de la pandémie de COVID-19. 
Mots-clés : apprentissage en ligne, enseignement supérieur, modèle éducatif, avenir de l'éducation, pandémie, Espagne, Catalogne

ABSTRACT

Albert Sangrà is full professor at the Open University of Catalonia (UOC) and director of the UNESCO Chair in Education and Technology for Social Change. As a founding member of this university, he briefly recalls its birth in the mid-1990s and emphasizes its particularity of offering fully online learning in the Catalan language that incorporated Spanish shortly afterward. He then outlines the principles on which e-learning is currently based at the UOC and, finally, shares his views on the future of this mode of training in light of the impact of the COVID-19 pandemic.

Keywords: online learning, higher education, educational model, future of education, pandemic, Spain, Catalonia

Albert Sangrà es Doctor en Pedagogía, investiga sobre los usos de las TIC en la educación y la formación y, en particular, sobre las políticas, la organización, la gestión, el liderazgo y la garantía de la calidad en la implementación de la educación en línea. Es investigador del grupo consolidado Edul@b y profesor catedrático de los Estudios de Psicología y Ciencias de la Educación. Actualmente es también el director del Programa de Doctorados Industriales de la Generalitat de Catalunya. Sus intereses de investigación se centran también en desarrollo profesional docente para la enseñanza en línea. Ha liderado y participado en numerosos proyectos de investigación, nacionales e internacionales. Es postgraduado por la Open University UK, y por Harvard University. Es consultor del World Bank. Ha sido galardonado con el Premio a la Excelencia en e-Learning 2015, otorgado por el World Education Congress, y también ha sido reconocido como EDEN Senior Fellow per l'European Distance and E-Learning Network. Recientemente ha coordinado la publicación del libro Decálogo para la mejora de la educación online. Propuestas para educar en contextos presenciales discontinuos.

MARCELO MAINA: Profesor Sangrà, ¿podría hablarnos del origen de la Universitat Oberta de Catalunya? En otras palabras, ¿en qué contexto y con qué objetivos surgió la idea de crear una universidad en línea?

ALBERT SANGRÀ: En la primera mitad de los años 90 existía en Cataluña una demanda para proveer educación a distancia de calidad en lengua catalana, puesto que en España la única universidad que ofrecía estudios a distancia era la UNED, y lo hacía solo en lengua castellana. El Gobierno de la Generalitat de Cataluña impulsó la creación de una universidad a distancia en catalán y encargó dicho proyecto al Dr. Gabriel Ferraté, académico de importante prestigio, que había sido Rector de la Universitat Politécnica de Cataluña durante 20 años.

El Dr. Ferraté imprimió al encargo una identidad muy marcada: a) no íbamos a crear una universidad a distancia, sino una universidad sin distancias; b) íbamos a hacer un uso intensivo de las tecnologías disponibles, y en aquel momento Internet era la piedra angular sobre la que debía descansar cualquier 
modelo que quisiera ser innovador; y c) la universidad debía tener una estructura organizativa ágil y flexible, que la hiciese capaz de responder a las demandas de la sociedad de manera adecuada e inmediata.

La UOC iba dirigida a un público mayormente adulto que, o bien no había tenido oportunidad de realizar sus estudios universitarios anteriormente, por razones varias, o bien los había empezado y no había podido finalizarlos, a su pesar. La idea fundacional de la UOC era ofrecer una formación universitaria de calidad y, a la vez, garantizar la máxima flexibilidad a los estudiantes: estudiar y aprender superando las limitaciones del espacio y del tiempo. Estudiar desde cualquier lugar, a cualquier hora, a su propio ritmo, garantizando la calidad de esta educación superior al nivel de cualquier universidad presencial. Palabras que ahora parecen lógicas y que todo el mundo suscribiría, pero que en aquel momento no parecían tan evidentes. La UOC fue la primera universidad que nació en la Sociedad de la Información y del Conocimiento, creada para la Sociedad de la Información y del Conocimiento.

\section{MARCELO MAINA: ¿Quiénes fueron los miembros que fundaron e iniciaron esta universidad? ¿Cómo llegaron a formar parte de esta?}

ALBERT SANGRÀ: La andadura de la universidad empezó con un grupo muy reducido de personas, alrededor de unas 10, entre las que se encontraban tanto el equipo rectoral -rectores y vicerrectores-, gerente, directores de área y personal de apoyo, como por supuesto, los primeros profesores y profesoras, en un número reducido, puesto que se decidió empezar poniendo en marcha una experiencia piloto con solo 200 estudiantes y dos titulaciones de ciclo corto que se iban desarrollando progresivamente: Empresariales y Psicopedagogía.

La universidad se dotó de aquellos equipos que podían poner en marcha todo el engranaje de una universidad no presencial, desde el diseño y elaboración de materiales y recursos de aprendizaje multimedia, hasta los sistemas tecnológicos de información y comunicación que tenían que aguantar el funcionamiento del Campus Virtual, el primero de su tipo y alcance en el mundo. Pasando, por supuesto, por las áreas académica, de recursos humanos y económica, y de relaciones con el entorno.

\section{MARCELO MAINA: ¿Puede describirnos cómo era la UOC en sus inicios? ¿Quiénes fueron los protagonistas principales y su modo de funcionamiento?}

ALBERT SANGRÀ: Desde el primer minuto, la UOC se concentró en servir a sus estudiantes. Todo el diseño del proceso de aprendizaje y los servicios académicos vinculados a él se llevó a cabo teniendo en cuenta que el estudiantado estaba en el centro de nuestro modelo, y era quien daba sentido a la existencia de la institución.

La UOC se creó con la mente puesta en su sostenibilidad, por lo que al cuerpo de docentes a tiempo completo que iba conformando la plantilla de profesorado de la universidad se les tenía que añadir el profesorado a tiempo parcial que ejercía de instructor o tutor en línea en cada una de las asignaturas. Así, los profesores a tiempo completo se responsabilizaban de los contenidos, de los criterios de desarrollo y de evaluación, mientras que los instructores asumían el apoyo a los estudiantes semestralmente. De esa forma, la institución podía asumir cambios en la magnitud del número de estudiantes sin que la calidad y el servicio que ofrecía se viesen mermados

Así, desde el primer día, al UOC fue una universidad enteramente virtual: todo se llevaba a cabo a través del Campus Virtual. 
MARCELO MAINA: Además del hecho de ser una universidad enteramente en línea, ¿cuáles cree que son las especificidades de la UOC en relación con otros establecimientos de educación superior españoles? ¿Cuáles son también sus peculiaridades en relación con otras instituciones internacionales especializadas en FAD como Athabasca University en Canada, Open University o el CNED?

ALBERT SANGRÀ: Primero, hay un elemento común que conviene destacar. La UOC es una universidad acreditada en España, lo que significa que es exactamente igual que cualquier otra universidad, sea presencial o no: tiene los mismos derechos y cumple las mismas obligaciones, y se somete periódicamente a la verificación y acreditación de los títulos que ofrece según establecen las normas ministeriales. Por lo tanto, sus títulos tienen exactamente la misma validez que los de cualquier universidad española y europea, al amparo del Espacio Europeo de Educación Superior.

Su particularidad es que se trata de una universidad en línea, que ofrece un mayor rango de flexibilidad a sus estudiantes, pero con exactamente el mismo rigor académico que cualquier otra universidad. Además, en la UOC la investigación ocupa un rol muy importante, pues la universidad siempre se ha definido como tal, llevando al máximo las tres misiones encomendadas a ella: enseñanza, investigación y transferencia de conocimiento a la sociedad. La UOC desarrolla la investigación disciplinaria que le corresponde en función de las áreas de conocimiento en las que ofrece formación, pero además, se especializa en tres grandes áreas interdisciplinares que marcan buena parte de su agenda estratégica en investigación y desarrollo: la Sociedad del Conocimiento, la e-Health y la educación en línea (o e-learning). Dispone de centros de investigación dedicados a ellas.

A nivel internacional, además de tener muy buena relación con las universidades que menciona, lo que nos ha permitido llevar a cabo proyectos de investigación conjuntos, hay algunos elementos que nos caracterizan. Probablemente, estamos más cerca de universidades como Athabasca o la Open University UK, porque compartimos un modelo en el sentido de ser universidades independientes que ofrecen las mismas titulaciones que las universidades presenciales, pero solo en la modalidad en línea. Creo que el CNED es un caso distinto, porque en Francia no funciona como universidad autónoma, sino que se coordina para trabajar con las universidades presenciales. Es una diferencia fundamental.

La UOC se caracteriza por un fuerte componente internacional, especialmente gracias al importante conjunto de estudiantes que provienen de países latinoamericanos. Además de la oferta en catalán, la UOC decidió el año 2000 doblar toda su oferta también en lengua castellana, con lo cual se amplió considerablemente el espectro de sus estudiantes potenciales. Esto, unido a la buena relación con las universidades de los países latinoamericanos, ha permitido llevar a cabo muchos proyectos de colaboración conjunta que han producido resultados muy provechosos para todas las partes.

MARCELO MAINA: ¿En qué medida los avances tecnológicos han transformado el proceso de creación de cursos en línea? ¿Cómo se han combinado las innovaciones educativas y tecnológicas a lo largo del tiempo?

ALBERT SANGRÀ: La UOC se creó hace 25 años y, por lo tanto, la tecnología ha avanzado mucho y ha permitido desarrollos que en aquel momento no nos podíamos ni imaginar. Sin embargo, creo que lo que más se ha avanzado ha sido la capacidad de conectar: los sistemas de telecomunicación han permitido que podamos pensar en recursos audiovisuales al alcance de ser usados por cualquier persona, en cualquier lugar.

Esto ha condicionado el tipo de recursos tecnológicos y debería condicionar los modelos pedagógicos subyacentes en el uso de esos recursos, puesto que se ha incrementado muchísimo la capacidad y 
posibilidad de interacción entre las personas y también la interactividad con los materiales. Disponemos de más facilidades en la gestión de los cursos, pero también podemos ofrecer más implicación (engagement) de los estudiantes en los mismos. Por otro lado, el desarrollo del movimiento "abierto" ha hecho que tendamos a reutilizar recursos ya existentes, sin que eso impida seguir elaborando nuevos recursos en diversidad de formatos y soportes.

Aunque lo ideal sería que cualquier innovación tecnológica estuviese vehiculada por sus beneficios pedagógicos, sabemos que eso no es así siempre, y eso sucede en todo el mundo. Especialmente, porque las innovaciones tecnológicas nunca se han creado pensando en la educación, sino que se intentan adaptar a ella. Por otro lado, existe una tendencia muy marcada, quizás demasiado, que hace que las innovaciones tecnológicas tiendan a replicar lo que se hace en la educación presencial, pensando que se trata de imitarla, cuando la educación a distancia y en línea tienen que basarse en sus propios principios pedagógicos.

\section{MARCELO MAINA: ¿Cómo definiría el modelo de profesorado de la UOC y la relación con el estudiantado? ¿Cómo ha evolucionado esta actividad a lo largo del tiempo?}

ALBERT SANGRÀ: En la UOC disponemos de varias figuras docentes. En primer lugar, los profesores responsables de asignatura (PRA) son los profesores-investigadores doctores de plantilla, expertos en determinadas áreas de conocimiento, los máximos responsables de cada una de las asignaturas que componen un programa o titulación. Llevan a cabo su misión a tiempo completo. Son responsables de su diseño, de la identificación y/o elaboración de materiales y recursos de aprendizaje necesarios para alcanzar los objetivos de la materia, y de los criterios de evaluación con los que se valorará el alcance de las competencias adquiridas por parte de los estudiantes. Además, deben supervisar el trabajo de los docentes colaboradores.

Los docentes colaboradores son especialistas en cada una de las asignaturas y son los que gestionan el plan docente que se presenta a los estudiantes al inicio de cada semestre académico. Son los que plantean las actividades a desarrollar, resuelven dudas, amplían recursos, y aplican los criterios de evaluación una vez que los estudiantes entregan cada una de las actividades que constituyen lo que llamamos "pruebas de evaluación continua".

Estos docentes tienen que asumir un rol de acompañamiento del estudiante, que es fundamental en nuestro modelo. Nuestros estudiantes deben asumir un rol activo y desarrollar su capacidad de autonomía y auto-regulación: deben resolver tareas, participar activamente en discusiones, llevar a cabo trabajos en grupo colaborativamente. Los docentes tienen que estar ahí orientándolos, guiándoles, ayudándoles a aclarar aquellos conceptos que no entienden suficientemente y facilitarles feedback de manera permanente, puesto que este es un elemento clave para la progresión en el aprendizaje de los estudiantes. Habitualmente, son profesores de otras universidades o profesionales del sector (en función de la titulación de la que se trate). Llevan a cabo su tarea a tiempo parcial, como colaboradores de la universidad. En función de la exigencia normativa, son doctores o disponen, como mínimo, del grado de Máster.

Existe otra figura no menos importante, que es la del profesor colaborador tutor. Este o esta docente actúa como un consejero, un asesor de los estudiantes a lo largo de toda su trayectoria en una misma titulación. Le orienta en el momento de decidir qué asignaturas matricular (la flexibilidad es máxima), le resuelve problemas con los que cualquier estudiante puede encontrarse a lo largo de años de estudio en línea y, en la última etapa, le asesora sobre posibles salidas profesionales y el contexto laboral que puede encontrar. 
El o la estudiante, por lo tanto, se encuentra en el mismo centro de un modelo que le plantea tener que desarrollar una serie de actividades que están diseñadas teniendo en cuenta las competencias que debe adquirir en el contexto del mapa competencial de una titulación determinada.

\section{MARCELO MAINA: ¿En qué medida han cambiado sus visiones y prácticas educativas durante su carrera?}

ALBERT SANGRÀ: No es una pregunta fácil de responder. Yo quizás diría que, más que cambiar, mis visiones se han ido consolidando a lo largo del tiempo a partir de evidencias que las han ido moldeando en la realidad. Por ejemplo, a lo largo de mi carrera siempre me han preguntado (de forma retórica, esperando una respuesta afirmativa) si hay disciplinas y enseñanzas que no se pueden llevar a cabo con la modalidad a distancia o en línea. Y, a medida que pasa el tiempo, tengo más claro que casi todo puede enseñarse y aprenderse en línea. Ahora bien, es cierto que eso dependerá del nivel de inversión económica que queramos o podamos aplicar, y de su necesidad. El uso cada vez más extendido de simulaciones, o de la realidad virtual, nos pone de manifiesto que casi todo es posible, pero a veces será muy caro y puede que no sea recomendable o sostenible. Pero posible, probablemente lo es.

Quizás el mayor cambio en las prácticas pedagógicas ha sido darme cuenta que los modelos tradicionales de educación a distancia, que se basaban en el acceso a los contenidos, han sido superados en cuanto a resultados de aprendizaje, por modelos en los que la interacción, la colaboración y la co-creación de conocimiento son más importantes que los propios contenidos, que cada vez son más accesibles. La importancia creciente de desarrollo de las competencias blandas, de saber qué buscar y dónde buscarlo y, sobre todo, para qué utilizarlo. Resolver problemas en lugar de acumular información o conocimientos. Ese creo que es el cambio sustancial en la educación de mi época. Y la tecnología ha ayudado sobremanera a ello.

MARCELO MAINA: La formación en línea ofrece a los alumnos y a los profesores una gran flexibilidad. Pone énfasis en su autonomía y la capacidad para regular sus actividades. A la luz de su experiencia, ¿tendría algún consejo para los profesores, o incluso para los estudiantes, que actualmente están descubriendo la formación en línea en este contexto de pandemia?

ALBERT SANGRÀ: Indiscutiblemente, la autonomía del estudiante y su capacidad de auto-regulación son elementos fundamentales para que una persona desarrollo con éxito un proceso de enseñanzaaprendizaje en la modalidad en línea. Los docentes tienen que ayudar a los estudiantes a desarrollar esas capacidades: es fundamental que aprendan a aprender, y que sean conscientes de que buena parte de su éxito depende de ellos mismos, de su propia ecología de aprendizaje y de la iniciativa que tomen para ser activos, propietarios de su propio proceso de aprendizaje.

Las instituciones tienen que poner a disposición de los estudiantes mecanismos para que adquieran o incrementen estas competencias, a las que debe añadirse la competencia digital -algo sustancial en pleno siglo XXI-. La UOC, por ejemplo, desde su creación, ha ofrecido una asignatura obligatoria en todas sus titulaciones que, específicamente, tenía como objetivo facilitar la adquisición de la competencia digital y de estudio entre los estudiantes. Era, y es, un instrumento fundamental para garantizar que puedan obtener buenos resultados en su proceso de aprendizaje.

Por otro lado, a los estudiantes les recordaría algo que probablemente ya saben o intuyen: la educación no presencial no es "no ir a clase". Aunque haya modelos, como el de la UOC, asíncronos, que ofrecen una flexibilidad superior a aquellos que exigen mucha sincronía, es fundamental que ellos se auto-exijan disciplina y responsabilidad. Una buena organización del estudio y un firme compromiso con los objetivos 
que quieren alcanzar son elementos fundamentales para una experiencia de aprendizaje satisfactoria. La flexibilidad es un atributo muy positivo siempre que se sepa gestionar bien. $Y$ eso también se puede aprender.

MARCELO MAINA: Para concluir, atendiendo al desarrollo de la formación a distancia, desde el auge de internet y con más fuerza desde el inicio de la pandemia, ¿qué futuro ve en el horizonte para la UOC y la formación en línea en general?

ALBERT SANGRÀ: Aunque soy de los que piensa que, desgraciadamente, muchas instituciones están esperando que la pandemia se supere definitivamente para volver otra vez a lo de antes, también es cierto que observo muchas otras instituciones que ya han decidido avanzar hacia la definición y diseño de nuevos modelos de enseñanza y aprendizaje híbridos, más flexibles y adaptables. Las decisiones que se tomen marcarán la diferencia entre las instituciones que volverán al pasado y las que avanzarán hacia el futuro. Desde un punto de vista estratégico, esta decisión va a ser crucial para muchas organizaciones.

Las instituciones presenciales continuarán existiendo, pero tendrán que definir muy bien cuál va a ser el valor añadido de la parte presencial de su oferta. Creo que la mayoría tenderán a proponer modelos híbridos, que tomarán formatos diversos, unos más exitosos que otros. Sin embargo, los estudiantes que busquen un grado de flexibilidad cercano al $100 \%$, no podrán asistir a todas estas instituciones. Y ese es el papel que continuarán desarrollando las universidades completamente en línea.

Durante la pandemia, la modalidad en línea ha sido la única opción para evitar la interrupción total de los sistemas educativos. Y, reconociéndolo o no, la mayor parte de instituciones presenciales ha tenido que observar cómo se puede enseñar y aprender en línea. Muchos lo han comprendido, aunque quizás no tantos como hubiésemos deseado, a la luz de algunas manifestaciones. En cualquier caso, las universidades en línea, como la UOC, como Athabasca, como la Open University o como TÉLUQ, están llamadas a seguir liderando el diseño de modelos de enseñanza y aprendizaje de máxima flexibilidad, de mayor personalización, y con el uso y aplicaciones de las tecnologías que permitan seguir garantizando la máxima interacción y colaboración entre estudiantes y profesores. $\mathrm{Y}$, por supuesto, garantizando una educación superior de alta calidad. 